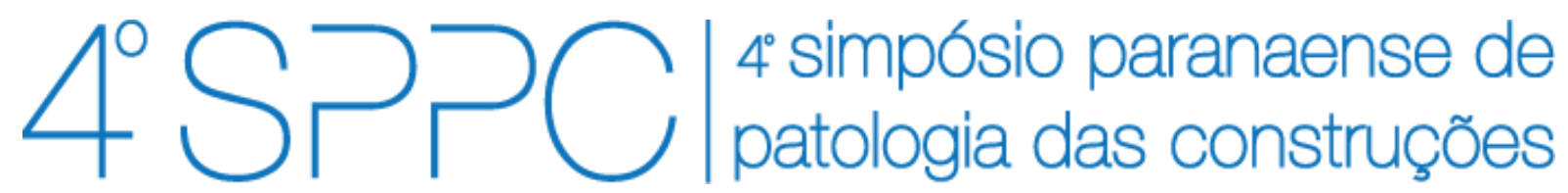

ISSN 2526-7248 artigo n. 4SPPC145, pp. 401-410, 2019

\title{
Influência de pozoalanas de alta reatividade no fator de idade do modelo de vida útil de Andrade (2004)
}

\author{
Real, Lígia Vitória ${ }^{1}$; Medeiros, Marcelo Henrique Farias ${ }^{2}$; Rivarola, Gustavo \\ Bortolan ${ }^{3}$ \\ ${ }^{1}$ Doutoranda, Engenheira Civil, USP, ligiavr@usp.br \\ 2 Professor Doutor, UFPR, medeiros.ufpr@gmail.com \\ ${ }^{3}$ Eng.ํㅡㄴ Civil, UFPR/PPGECC, gustbrv@gmail.com
}

\begin{abstract}
Resumo: A preocupação com a construção de estruturas mais sustentáveis e o crescimento dos custos de manutenção têm motivado a comunidade científica a desenvolver modelos de estimativa de vida útil de estruturas de concreto. Neste trabalho estudam-se adições de pozolanas de alta reatividade, restringindo-se à sua influência sobre o fator de idade (q) do modelo proposto por Andrade (2004) ao caso de ataque por íons cloreto. Neste modelo, a resistividade elétrica superficial do concreto (RES) é o parâmetro fundamental, onde o fator de idade indica o quanto a RES irá crescer ao longo do tempo. Três diferentes adições ao traço de referência foram testadas, a saber: sílica ativa, sílica de casca de arroz e metacaulim - com o objetivo de analisar suas influências sobre "q" e seu desenvolvimento ao longo do tempo. Observou-se incremento de "q" bem como de RES sobre o traço de referência. Observou-se redução de "q" ao longo do tempo para os traços de sílica ativa e de sílica de casca de arroz. Já o traço de referência e o de metacaulim apresentaram tendência de estabilização de "q" ao longo do tempo.
\end{abstract}

Palavras-chave: modelo de vida útil de estruturas de concreto, adições minerais de alta reatividade pozolânica, fator de idade, resistividade elétrica superficial.

\begin{abstract}
The concern about construction of more sustainable structures and the growth of maintenance costs have motivated the scientific community to develop models of service life estimation for concrete structures. In this paper, additions of pozzolans of high reactivity are being studied, restricted to their influence on the age factor (q) of the model proposed by Andrade (2004) to the case of chloride ions attack. In this model, the surface electrical resistivity of the concrete (SER) is the fundamental parameter, where "q" indicates how much SER will grow over time. Three different additions to the reference mix were tested, namely: active silica, rice husk silica and metakaolin - aiming to analyze their influence on " $q$ " and its development over time. Increase of " $q$ " as well as of SER over the reference mix was shown. "q" reduction was observed over time for the mixes of active silica and rice husk silica. However, the baseline mix and metakaolin showed a tendency to stabilize "q" over time.
\end{abstract}

Keywords: service life model of concrete structures, high pozzolanic reactivity mineral additions, age factor, surface electrical resistivity. 
REAL, L. V.; MEDEIROS, M. H. F.; RIVAROLA, G. B. INFLUÊNCIA DE POZOLANAS DE ALTA REATIVIDADE NO FATOR DE IDADE DO MODELO DE VIDA ÚTIL DE ANDRADE (2004). $4^{\circ}$ Simpósio Paranaense de Patologia das Construções (40 SPPC), artigo 4SPPC145, pp. 401 - 410, 2019. DOI: 10.4322/2526-7248.062

\section{Introdução}

O crescimento de custos com manutenção e recuperação de estruturas de concreto têm despertado o interesse na questão da durabilidade. No Brasil, a norma de desempenho estabelece uma vida útil de projeto mínima de 50 anos. Visando atender essa necessidade, as normas NBR 12655 [1] e NBR 6118 [2] estabelecem critérios relacionados à classe de agressividade ambiental, ao cobrimento mínimo de armadura, à composição do traço (consumo mínimo de cimento e máxima relação água/aglomerante), ao limite de abertura de fissuras, às formas arquitetônicas e aos dispositivos de drenagem, como por exemplo, a presença de pingadeiras.

A fim de quantificar a vida útil das estruturas de concreto armado sujeitas a corrosão na presença de cloretos, Andrade [3] desenvolveu um modelo de vida útil que tem como principal parâmetro a resistividade elétrica superficial. $O$ método foi desenvolvido e vem sendo estudado pelo grupo de pesquisa de durabilidade do concreto do Instituto de Ciência da Construção Eduardo Torroja, em Madrid, na Espanha. Recentemente, o modelo foi aplicado para o desenvolvimento do concreto para duplicação do Canal do Panamá, pois as autoridades responsáveis pela ampliação exigiram vida útil de 100 anos para os elementos estruturais de concreto armado [4].

A norma UNE 83988-2 [5] define a Resistividade Elétrica Superficial (RES) do concreto saturado como uma medida indireta de conectividade e tamanho dos poros e para um concreto não saturado, indica o grau de saturação do material. Ou seja, a RES depende da estrutura de poros do concreto e da composição química da solução aquosa que os preenche. A continuidade dos poros assegura a movimentação de carga elétrica no concreto. [6]. Como o uso de adições pozolânicas gera refinamento e melhor distribuição dimensional de poros, bem como reduz a quantidade de íons presente na solução dos poros, contribui para a redução da condutividade elétrica, causando incremento direto na RES [7].

A influência das adições minerais no traço já foi observada em comparações de RES dos cimentos brasileiros CPIV32, CPIII40RS, CPIIF32 e CPV ARI no estudo de Medeiros $\mathrm{Jr}$ et al. [8], onde os resultados apontaram as maiores RES para as amostras do CP III 40 RS, com alto teor de escória de alto forno. Santor [9] também avaliou que provavelmente a adição mineral contribui com a redução da condutividade através do refinamento dos poros do concreto, refletindo em um aumento da RES medida.

Neste contexto apresentado, o presente trabalho tem por objetivo avaliar a influência da sílica ativa, metacaulim e sílica de casca de arroz no fator de idade, necessário para o emprego do modelo de vida útil de Andrade (2004).

\subsection{Modelo de previsão de vida útil proposto por Andrade (2004)}

O tempo de vida útil considerando o processo de corrosão em estruturas de concreto armado pode ser dividido em duas partes: iniciação e propagação. $O$ período de iniciação ou de despassivação ocorre a partir do momento em que o agente agressivo penetra na superfície do concreto até o início da corrosão. $O$ período de propagação consiste no desenvolvimento do processo corrosivo até o 
fim do grau aceitável desse fenômeno [10]. O Modelo de Andrade leva em consideração os dois períodos denominados: ti e tp. Entretanto este trabalho trata apenas do tempo de iniciação. De acordo com Andrade [3] pode-se considerar ti, o tempo de iniciação, como critério limite de vida útil assumindo a forma da Equação 1.

$$
t_{i}=\frac{x^{2} \cdot \rho_{e f} \cdot\left(\frac{t}{t_{0}}\right)^{q}}{K_{C l}} \cdot r_{C l}
$$

Onde: $t_{i}=$ tempo de iniciação (em anos); $x=$ profundidade de penetração da concentração limite de cloretos (pode ser considerado como o recobrimento da armadura); $\rho_{\text {ef }}=$ resistividade elétrica superficial da primeira medição realizada para determinação do fator de idade (q); to é a idade de cura do concreto quando da primeira tomada de medição de RES utilizada para determinação de $q$; $t$, por sua vez é a correspondente idade quando da útima medição de RES; q = fator de idade; $r_{c l}=$ fator de reação; e $K_{c l}=$ fator ambiental.

Foco do presente trabalho, o fator de idade representa o progresso da hidratação do cimento ao longo do tempo, o refinamento dos poros e o consequente aumento da RES (ANDRADE \& D'ANDREA [11]; MEDEIROS JR. [8]). É calculado através da Equação 2, a qual permite observar sua íntima ligação à $R E S$, representando 0 quanto ela ainda será aumentada no decorrer do tempo.

$$
\rho_{t}=\rho_{0} \cdot\left(\frac{t}{t_{0}}\right)^{q}
$$

Onde: $\rho_{\mathrm{t}}=$ resistividade elétrica em qualquer idade; $\rho_{0}=$ resistividade elétrica na primeira medição; t é a idade da última medição; $t_{0}$ é a idade da primeira medição e q é o fator de idade.

Graficamente o fator de idade pode ser obtido diretamente pela inclinação da curva de regressão, plotada em escala logarítmica, do inverso da resistividade em função do tempo, onde - $q$ é o expoente da equação (ANDRADE \& D'ANDREA [11]).

\section{Programa experimental}

Visando avaliar do efeito de adições minerais de alta reatividade 403ozolânicas foram realizadas medidas de RES pelo método de Wenner em quatro diferentes traços descritos na Tabela 1.

Tabela 1: Traços unitários em massa

\begin{tabular}{lcccccccc}
\hline Traço & $\begin{array}{c}\text { CPV } \\
\text { ARI }\end{array}$ & $\begin{array}{c}\text { Sílica } \\
\text { Ativa }\end{array}$ & $\begin{array}{c}\text { Sílica de } \\
\text { Casca de } \\
\text { Arroz }\end{array}$ & Metacaulim & Areia & $\begin{array}{c}\text { Brita } \\
\mathbf{1}\end{array}$ & a/a & $\begin{array}{c}\text { Aditivo } \\
\text { (\%) }\end{array}$ \\
\hline TCPV & 1 & - & - & - & 2,25 & 3 & 0,45 & 1,8 \\
TSA & 0,9 & 0,1 & - & - & 2,25 & 3 & 0,45 & 1,8 \\
TSCA & 0,9 & - & 0,1 & - & 2,25 & 3 & 0,45 & 1,3 \\
TM & 0,9 & - & - & 0,1 & 2,25 & 3 & 0,45 & 1,9 \\
\hline \multicolumn{7}{c}{ a/a - relação água/aglomerante } \\
& \multicolumn{7}{c}{}
\end{tabular}


REAL, L. V.; MEDEIROS, M. H. F.; RIVAROLA, G. B. INFLUÊNCIA DE POZOLANAS DE ALTA REATIVIDADE NO FATOR DE IDADE DO MODELO DE VIDA ÚTIL DE ANDRADE (2004). $4^{\circ}$ Simpósio Paranaense de Patologia das Construções (40 SPPC), artigo 4SPPC145, pp. 401 - 410, 2019. DOI: 10.4322/2526-7248.062

As composições das misturas partiram do traço de referência (TCPV), sem qualquer tipo de adição. Os traços com pozolanas tiveram $10 \%$ do cimento, em massa, substituído pelos respectivos materiais cimentícios suplementares a saber: sílica ativa, sílica de casca de arroz e metacaulim.

O consumo de cimento foi padronizado em $360 \mathrm{~kg} / \mathrm{m}^{3}$, o fck mínimo em 40 Mpa e a relação água/aglomerante em 0,45 , o que atende aos parâmetros de garantia mais rígidos quanto à classe IV de agressividade descritos na norma da ABNT NBR12655 [1]. As Tabelas 2 e 3 mostram a caracterização física e química do cimento utilizado neste programa experimental.

Tabela 2: Características físicas do CPV ARI

\begin{tabular}{|c|c|c|c|c|c|c|c|c|}
\hline \multirow{2}{*}{$\begin{array}{c}\text { Massa } \\
\text { Específica } \\
\left(\mathbf{g} / \mathbf{c m}^{3}\right) \\
\end{array}$} & \multicolumn{2}{|c|}{ Tempo de pega } & \multirow[t]{2}{*}{$\begin{array}{c}\text { BET } \\
\left(\mathrm{m}^{2} / \mathrm{g}\right)\end{array}$} & \multicolumn{4}{|c|}{$\begin{array}{c}\text { Resistência à } \\
\text { compressão (Mpa) }\end{array}$} & observação \\
\hline & Início & Fim & & 1d & $3 d$ & $7 d$ & 28d & \multirow{2}{*}{$\begin{array}{l}\text { adição } \leq 5 \% \\
\text { filer calcário }\end{array}$} \\
\hline 3,12 & $2 \mathrm{~h} 47$ & $3 \mathrm{~h} 23$ & 1,070 & 22 & 37,9 & 43,3 & 51,15 & \\
\hline
\end{tabular}

Dentre os cimentos fabricados no estado do Paraná, o CPV ARI é o mais puro, pois contém o menor teor de adições, observado na Tabela 2 - por esta razão foi escolhido para compor os traços estudados nesta pesquisa.

Tabela 3: Características químicas do CPV ARI

\begin{tabular}{cccccc}
\hline $\mathrm{SiO}_{2}$ & $\mathrm{Fe}_{2} \mathrm{O}_{3}$ & $\begin{array}{c}\text { Características Químicas (\%) } \\
\mathrm{CaO}\end{array}$ & $\mathrm{SO}_{3}$ & $\mathrm{MgO}$ & $\mathrm{Al}_{2} \mathrm{O}_{3}$ \\
\hline 18,63 & 2,63 & 60,64 & 2,85 & 4,83 & 4,33 \\
\hline $\begin{array}{c}\text { Perda ao } \\
\text { fogo }\end{array}$ & $\mathrm{CaO}$ livre & $\begin{array}{c}\text { Resíduo } \\
\text { Insolúvel }\end{array}$ & $\begin{array}{c}\text { Equivalente } \\
\text { alcalino }\end{array}$ & Teor de $\mathrm{C}_{3} \mathbf{A}$ \\
\hline 2,89 & 0,92 & 0,66 & 0,61 & \multicolumn{2}{c}{7,00} \\
\hline
\end{tabular}

Foi utilizado aditivo superplastificante de terceira geração com o objetivo de se obter a mesma trabalhabilidade em todos os traços. Os agregados utilizados foram areia natural de quartzo e brita 1 de origem granítica.

O critério de escolha das adições cimentícias suplementares priorizou as de alta reatividade 404 ozolânicas. Apresentam-se nas Tabelas 4 e 5 os resultados dos ensaios de caracterização das adições minerais. Foram realizados os ensaios: Chapelle modificado [12], análise química através da fluorescência de raios X; massa específica [13] e área específica pelo método de BET.

Tabela 4: Massa específica e atividade pozolânica das adições

\begin{tabular}{lccc}
\hline \multicolumn{1}{c}{$\begin{array}{c}\text { Adição } \\
\text { Mineral }\end{array}$} & $\begin{array}{c}\text { Massa Específica } \\
\left(\mathbf{g} / \mathbf{c m}^{3}\right)\end{array}$ & $\begin{array}{c}\text { BET } \\
\left(\mathbf{m}^{\mathbf{2}} \mathbf{g} \mathbf{)}\right.\end{array}$ & $\begin{array}{c}\text { Chapelle Modificado [12] } \\
\left(\mathbf{m g ~ C a}(\mathbf{O H})_{2} / \mathbf{g} \text { amostra }\right)\end{array}$ \\
\hline Metacaulim & 2,5 & 23,258 & 1193 \\
$\begin{array}{l}\text { Sílica ativa } \\
\begin{array}{l}\text { Sílica de } \\
\text { casca de }\end{array}\end{array}$ & 2,18 & 20,238 & 1542 \\
arroz & 2,12 & 14,692 & 1336 \\
\hline
\end{tabular}


REAL, L. V.; MEDEIROS, M. H. F.; RIVAROLA, G. B. INFLUÊNCIA DE POZOLANAS DE ALTA REATIVIDADE NO FATOR DE IDADE DO MODELO DE VIDA ÚTIL DE ANDRADE (2004). $4^{\circ}$ Simpósio Paranaense de Patologia das Construções (40 SPPC), artigo 4SPPC145, pp. $401-410$, 2019. DOI: 10.4322/2526-7248.062

Tabela 5: Análise química por fluorescência de raios $X$ das adições minerais

\begin{tabular}{lccccccccc}
\hline \multicolumn{1}{c}{ Adição } & & \multicolumn{8}{c}{ Composição Química (\%) } \\
\multicolumn{1}{c}{ Mineral } & $\mathbf{S i O}_{\mathbf{2}}$ & $\mathbf{F e}_{2} \mathbf{O}_{3}$ & $\mathbf{C a O}$ & $\mathbf{S O}_{3}$ & $\mathbf{A l}_{2} \mathbf{O}_{3}$ & $\mathbf{P}_{2} \mathbf{O}_{6}$ & $\mathbf{T i O}_{2}$ & $\mathbf{K}_{2} \mathbf{O}$ & $\mathbf{M g O}$ \\
\hline $\begin{array}{l}\text { Metacaulim } \\
\text { Sílica ativa }\end{array}$ & 57,8 & 3,9 & 0,14 & 1,63 & 46,05 & - & 2,22 & - & - \\
$\begin{array}{l}\text { Sílica de } \\
\text { casca de } \\
\text { arroz }\end{array}$ & 88,49 & 0,13 & 0,51 & 2,08 & 1,91 & - & - & 2,76 & - \\
\hline
\end{tabular}

\subsection{Ensaio de resistividade elétrica superficial}

Conforme mencionado anteriormente, o método utilizado para avaliar as RES dos diferentes traços foi o de Wenner. Nesse ensaio, a resistividade elétrica superficial da amostra é obtida através da medição da voltagem decorrente da indução de uma corrente conhecida através do corpo de prova. A Figura 2 mostra um esquema do equipamento de medição de RES pelo método de Wenner que segue o procedimento da norma UNE 83988-2 [5].

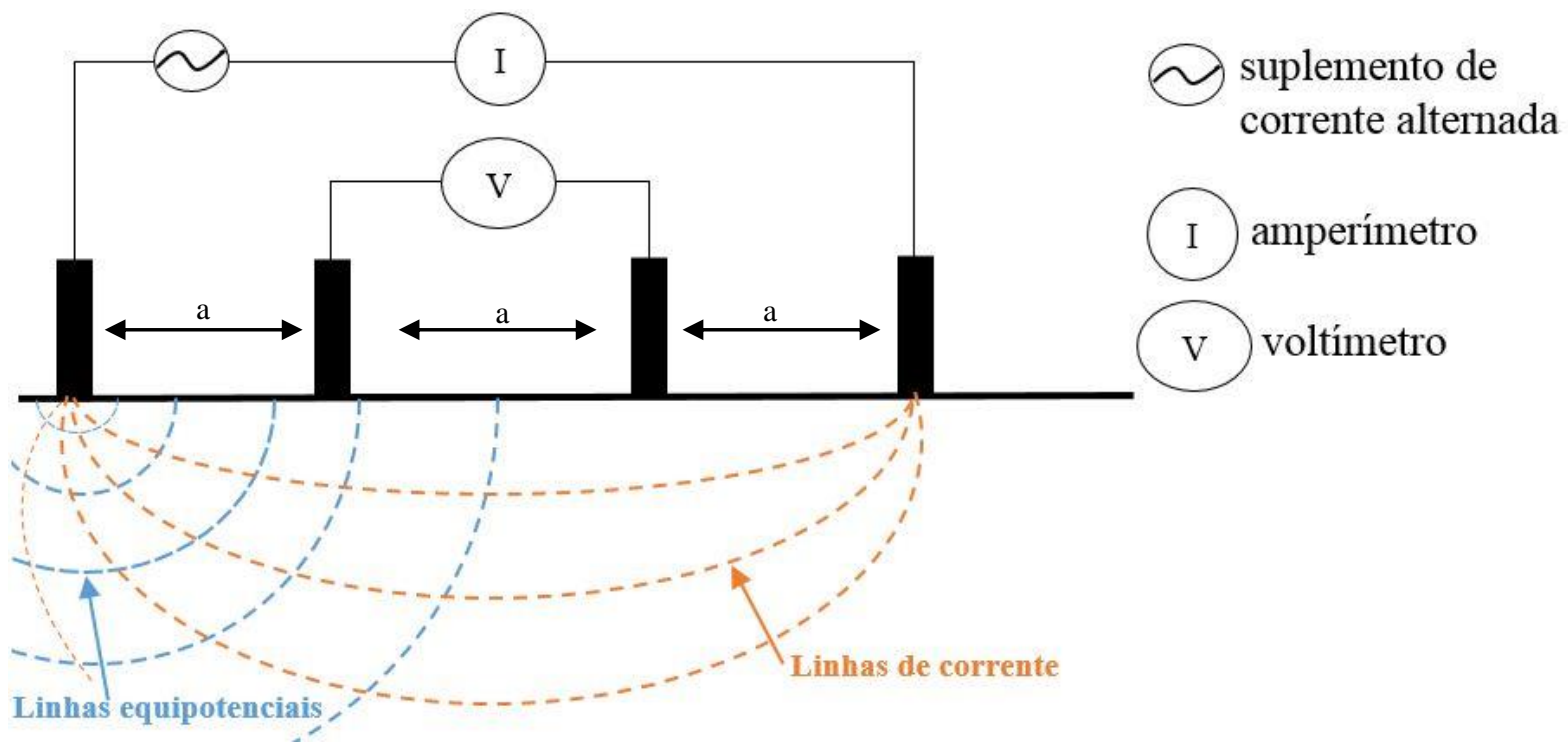

Figura 2: Esquema da Sonda Wenner (adaptado de MEDEIROS, [14])

A indução da corrente e leitura da voltagem são feitas com o auxílio de um equipamento que possui quatro eletrodos alinhados a uma distância fixa (a) conhecida, que em contato com o corpo de prova imprime uma corrente conhecida entre os dois eletrodos externos. A voltagem consequente é captada pelos eletrodos internos [14]. O espaçamento (a) entre os eletrodos do equipamento de medição de RES foi igual a $50 \mathrm{~mm}$, frequência igual a $40 \mathrm{~Hz}$ e corrente variando entre 10 a $200 \mu \mathrm{A}$. Até o momento das leituras, as amostras permaneceram em câmara úmida, garantindo saturação do concreto conforme requisito da UNE 83988-2 [5]. As medições foram realizadas nas idades de 7, 28, 56, 91, 154, 273, 364 e 400 dias de idade. 
REAL, L. V.; MEDEIROS, M. H. F.; RIVAROLA, G. B. INFLUÊNCIA DE POZOLANAS DE ALTA REATIVIDADE NO FATOR DE IDADE DO MODELO DE VIDA ÚTIL DE ANDRADE (2004). $4^{\circ}$ Simpósio Paranaense de Patologia das Construções (40 SPPC), artigo 4SPPC145, pp. 401 - 410, 2019. DOI: 10.4322/2526-7248.062

Observando as recomendações de Medeiros [14], Gowers \& Millard [15], Lencioni \& Lima [16], optou-se por moldar cubos de aresta de $250 \mathrm{~mm}$, maiores do que o prescrito na norma europeia a fim de simular um meio semi-infinito e consequentemente não haver a influência da geometria da peça sobre a leitura de resistividade. Foram moldados 5 corpos de prova por amostra, sendo tomadas 6 medidas, uma em cada face, totalizando 30 leituras por traço.

\section{Resultados e discussões}

\subsection{Resistividade elétrica superficial}

Apresentam-se na Figura 3 os resultados de RES ao longo do tempo para cada pozonala avaliada. Depois da sílica ativa, a sílica de casca de arroz foi a pozolana que mostrou maior capacidade de elevação da resistividade elétrica do concreto. $O$ traço referencia com cimento CPV ARI foi o que menos aumentou ao longo do tempo, tendo desenvolvido sua capacidade de reação praticamente completa nas primeiras idades. Todas as 3 pozolanas testadas apresentaram capacidade de aumentar a resistividade do concreto, indicando serem adições que dificultam o avanço do processo de corrosão, caso ela seja iniciada.

Todas as linhas de tendência de crescimento logarítmico apresentaram coeficientes de determinação $\left(\mathrm{R}^{2}\right)$ maiores que 0,90 . Comparando todas as adições testadas, 0 crescimento mais acentuado da RES foi observado para o traço com adição de sílica ativa. As barras de erro representam o desvio padrão de uma população de 30 dados.

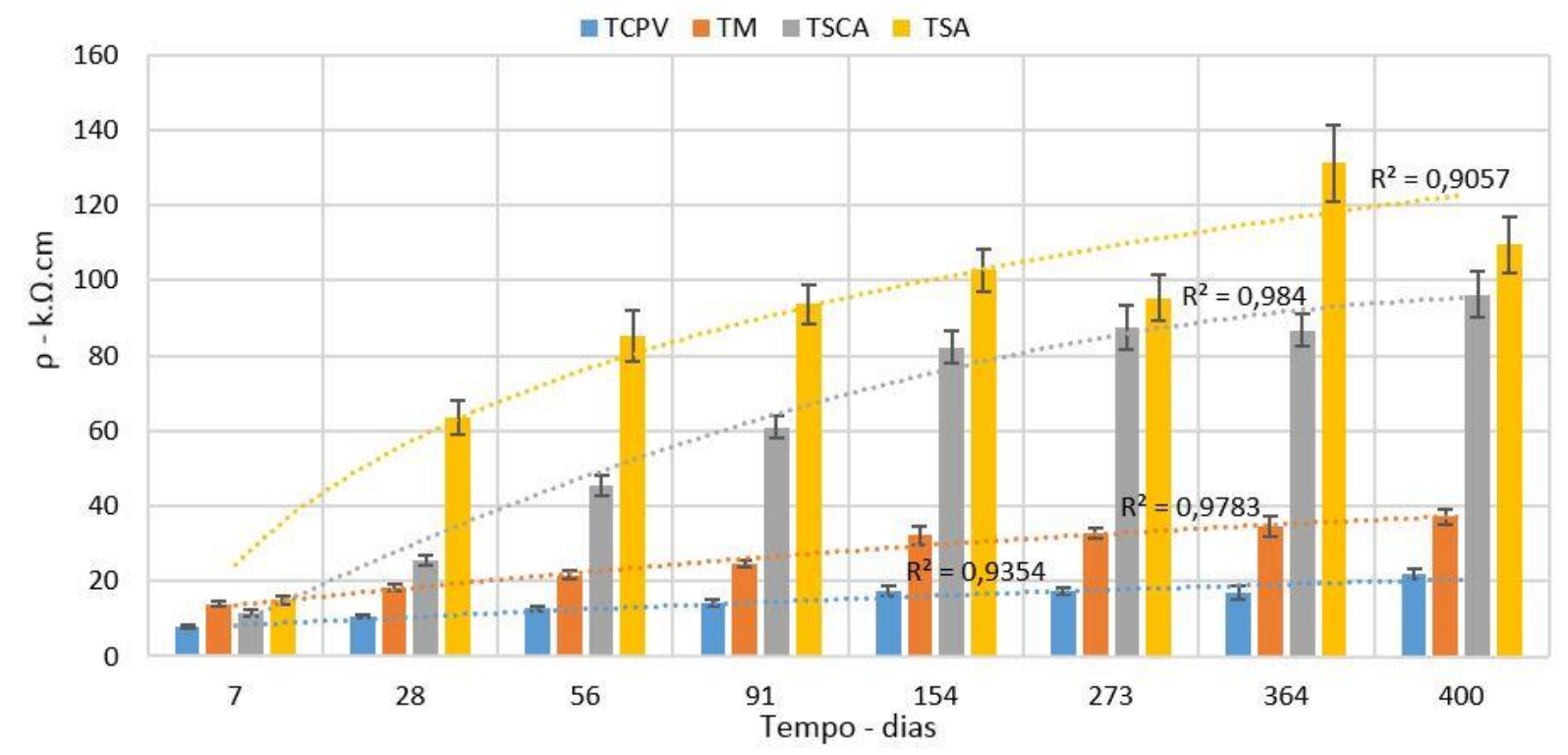

Figura 3: Evolução da RES ao longo do tempo em função da influência das adições pozolanicas testadas 
REAL, L. V.; MEDEIROS, M. H. F.; RIVAROLA, G. B. INFLUÊNCIA DE POZOLANAS DE ALTA REATIVIDADE NO FATOR DE IDADE DO MODELO DE VIDA ÚTIL DE ANDRADE (2004). $4^{\circ}$ Simpósio Paranaense de Patologia das Construções (40 SPPC), artigo 4SPPC145, pp. 401 - 410, 2019. DOI: 10.4322/2526-7248.062

\subsection{Fator de idade}

A Figura 4 exemplifica para o traço TSCA, a representação gráfica para diferentes idades de cura (t), respectivamente iguais a $91,154,273,364$ e 400 dias. Todos os cálculos foram feitos tomando-se a idade da primeira leitura de RES em $t_{0}=28$ dias.

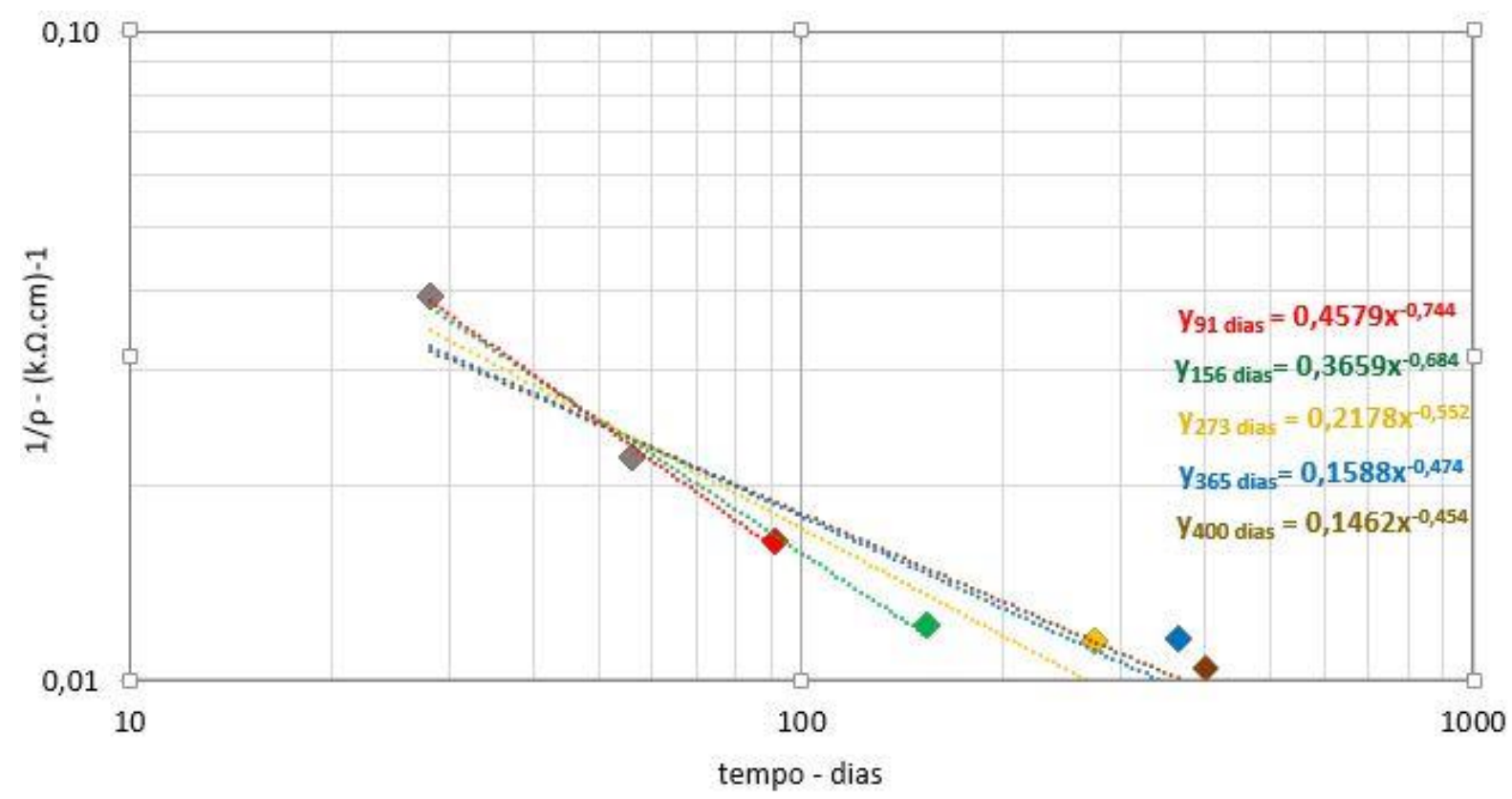

Figura 4: Representação gráfica do fator de idade TSCA

Para os traços em estudo, a Figura 5 representa a evolução dos valores de "q" obtidos para as diversas idades de cura.

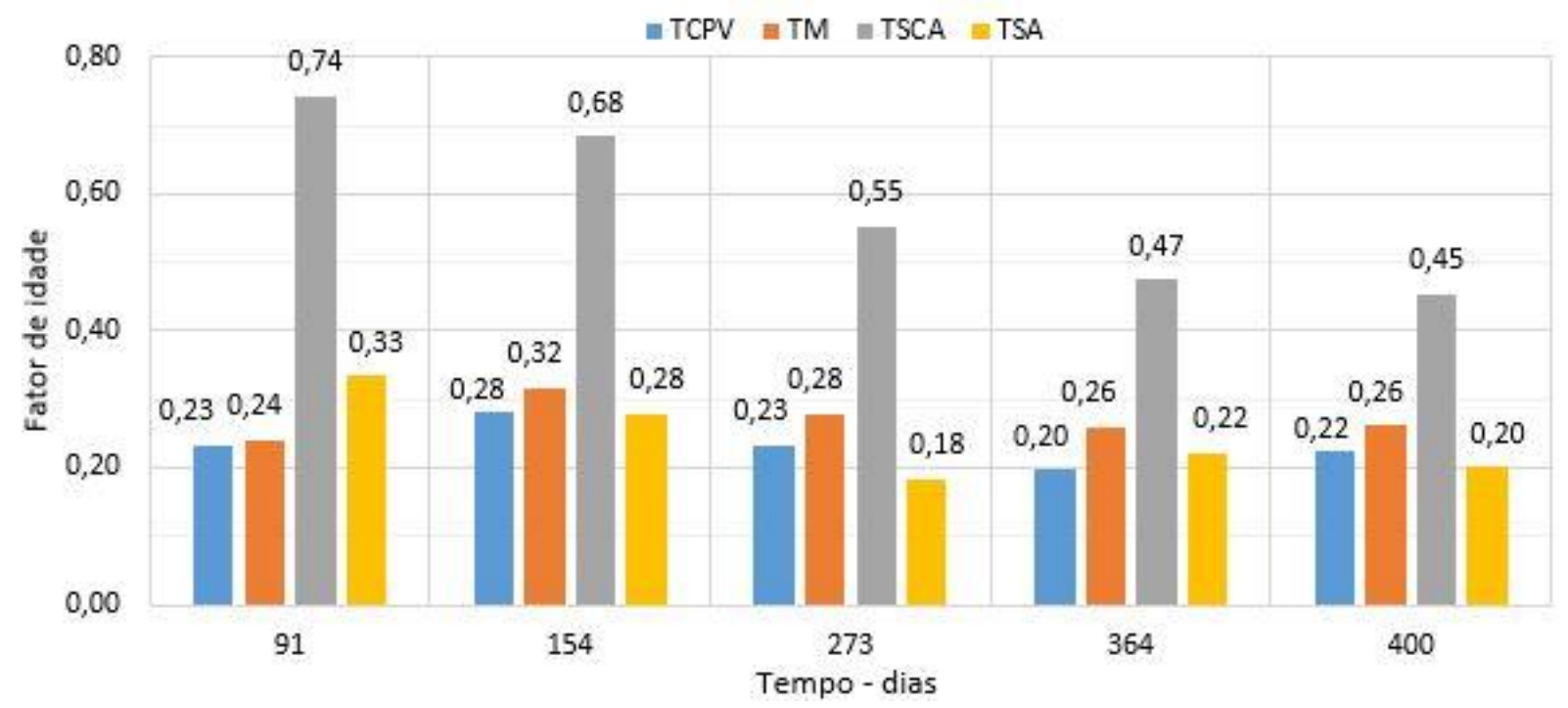

Figura 5: Evolução do fator de idade por traço 
REAL, L. V.; MEDEIROS, M. H. F.; RIVAROLA, G. B. INFLUÊNCIA DE POZOLANAS DE ALTA REATIVIDADE NO FATOR DE IDADE DO MODELO DE VIDA ÚTIL DE ANDRADE (2004). $4^{\circ}$ Simpósio Paranaense de Patologia das Construções (40 SPPC), artigo 4SPPC145, pp. 401 - 410, 2019. DOI: 10.4322/2526-7248.062

Observa-se a tendência de redução do fator de idade para os traços com as adições de sílica ativa e de sílica de casca de arroz com a tendência mais acentuada para este último. Por outro lado, para o traço de referência e para o traço com adição de metacaulim ocorreu uma estabilidade de resultados com leve tendência de crescimento aos 154 dias.

O traço de referência apresentou o menor dentre todos os valores do fator de idade. Pelo fato do CPV-ARI possuir alta finura, alta superfície específica e reatividade, as reações de hidratação ocorrem de maneira mais rápida, justificando-se os valores obtidos.

As adições minerais, como observado nos resultados, tendem a aumentar o fator de idade do concreto, porém observa-se que isso não acontece com o metacaulim. Por se tratar de uma adição mineral com teor de $46 \%$ de $\mathrm{Al}_{2} \mathrm{O}_{3}$, rica em alumínio, ela reage muito rapidamente com o hidróxido de cálcio presente na mistura formando aluminatos de cálcio hidratados [17], [18]. O traço TSA também apresentou baixo fator idade, provavelmente pela sílica ativa ser a adição mais reativa dentre as demais no presente estudo. Assim as reações ocorreram de forma mais intensa logo nas primeiras idades. Paralelamente, observa-se que este traço se destacou por apresentar elevada resistividade já aos 28 dias.

Deve-se observar que de acordo com a Equação 1, quanto maior o fator de idade, maior o tempo de iniciação estimado de acordo com Andrade [3]. Isso significa que os dados deste trabalho mostram que a adição de sílica de casca de arroz foi a adição que teve maior contribuição do fator de idade para aumento do tempo de iniciação do concreto armado.

\section{Conclusões}

Este artigo apresenta como considerações finais:

- A resistividade elétrica superficial foi influenciada pelo uso de adições pozolânicas no concreto, sendo as séries organizadas em ordem crescente de RES: TCPV $<$ TM $<$ TSCA $<$ TSA;

- A sílica de casca de arroz apresentou os maiores valores de fator idade entre as séries testadas neste experimento. Esta adição indicou uma redução do fator de idade ao longo do tempo de cura, mostrando que este fator de entrada no modelo vida útil pode sofrer alterações em função do tempo usado para seu cálculo;

- As séries de referência com metacaulim apresentaram tendência de aumento do fator de idade até 154 dias e a partir de 273 dias mostrou estabilidade deste parâmetro. 
REAL, L. V.; MEDEIROS, M. H. F.; RIVAROLA, G. B. INFLUÊNCIA DE POZOLANAS DE ALTA REATIVIDADE NO FATOR DE IDADE DO MODELO DE VIDA ÚTIL DE ANDRADE (2004). $4^{\circ}$ Simpósio Paranaense de Patologia das Construções (40 SPPC), artigo 4SPPC145, pp. $401-410$, 2019. DOI: 10.4322/2526-7248.062

\section{Referências}

[1] Associação brasileira de normas técnicas. NBR 12655: Concreto de cimento Portland - preparo, controle, recebimento e aceitação - Procedimento. Rio de Janeiro, 2015.

[2] Associação brasileira de normas técnicas. NBR 6118: Projetos de estruturas de concreto - Procedimento. Rio de Janeiro, 2014.

[3] Andrade, C. Calculation of initiation and propagation periods of service-life of reinforcements by using the electrical resistivity. International Symposium on Advances in Concrete through Science and Engineering, RILEM Symposium, Evanston, 2004.

[4] Andrade, C.; Rebolledo, N.; Castillo, A.; Tavares, F.; Pérez, R.; BAZ. M. Evaluación de mezclas de hormigón para el nuevo canal de Panamá mediante la medida de la resistividad y la resitencia a la difusión de cloruros. XII Congreso Latinoamericano de Patología de la Construcción y XIV Congreso de Control de Calidad en la Construcción, Colombia, 2013.

[5] Associación española de normalización e certificación. UNE 83988-2: Durabilidade del hormigón - métodos de ensayo - determinación de la resistividad elétrica - parte 2: método de las cuatro puntas o de Wenner. Madrid, 2014.

[6] Brameschuber, W.; Raupach, M.; Schröeder, P.; Dauberschmidt, C. Nondestructive determination of the water-content in the concrete cover using the multiring-electrode. International Symposium Non-destructive Testing in Cvivil Engineering, Berlin, 2003.

[7] Hoppe, T. F. Resistividade elétrica de concretos contendo diferentes teores de cinza de casca de arroz. Dissertação (mestrado). Universidade Federal de Santa Maria, 2005.

[8] Medeiros JR., R. A.; Lima, M. G.; Medeiros, M. H. F.; Real, L. V. Investigação da resistência à compressão e da resistividade elétrica de concretos com diferentes tipos de cimento. Revista ALCONPAT, 2014.

[9] Santor, M. S. Influência de materiais de proteção superficial na resistividade elétrica e na absorção capilar do concreto. Dissertação (mestrado), Universidade Federal de Santa Maria, Santa Maria, 2011.

[10] Torres, A. S. Corrosão por cloretos em estruturas de concreto armado: uma meta-análise. Tese (doutorado), Universidade Federal do Rio Grande do Sul, Escola de Engenharia, Programa de Pós Graduação em Engenharia Civil, Porto Alegre, 2011.

[11] Andrade, C.; D'andrea, R. Electrical resistivity as microstructural parameter for the modelling of service life of reinforced concrete structures. $2^{\text {nd }}$ International symposium on service life design for infrastructure. Deft, 2010.

[12] Associação brasileira de norma técnicas. NBR 15895: Materiais pozolânicos Determinação do teor de hidróxido de cálcio fixado - Método Chapelle modificado. Rio de Janeiro, 2010. 
REAL, L. V.; MEDEIROS, M. H. F.; RIVAROLA, G. B. INFLUÊNCIA DE POZOLANAS DE ALTA REATIVIDADE NO FATOR DE IDADE DO MODELO DE VIDA ÚTIL DE ANDRADE (2004). $4^{\circ}$ Simpósio Paranaense de Patologia das Construções (40 SPPC), artigo 4SPPC145, pp. 401 - 410, 2019. DOI: 10.4322/2526-7248.062

[13] Associação brasileira de normas técnicas. NBR 16605: Cimento Portland e outros materiais em pó - Determinação da massa específica - Procedimento. Rio de Janeiro, 2017.

[14] Medeiros, M. H. F. Estudo de variáveis que influenciam nas medidas de resistividade de estruturas de concreto armado. Revista Engenharia Civil da Universidade do Minho, v.12, Guimarães, 2001.

[15] Gowers, K. R; Millard, S. G. Measurement of concrete resistivity for assessment of corrosion severity of steel using wenner technique. ACl material Journal, v. 96-M66, p. 536-541, 1999.

[16] Lencioni, J. W.; Lima, M. G de. Principais fatores intervenientes nas medidas de resistividade elétrica superficial do concreto - estado-da-arte. VI Congresso Internacional sobre Patologia e Reabilitação de Estruturas, Córdoba, 2010.

[17] Ambroise, J.; Maximilien, S.; pera, J. Properties of metakaolin blended cements. Advanced Cement Based Materials, v.1(4), p. 161-168, 1994.

[18] Bartata, M. S. Concreto de alto desempenho no estado do Pará: estudo da viabilidade técnica e econômica de produção de concreto de alto desempenho com materiais disponíveis em Belém através do emprego de adições de sílica ativa e metacaulim. Dissertação (mestrado). Universidade Federal do Rio Grande do Sul, Porto Alegre, 1998. 